\title{
REPRESENTATION THEOREMS FOR SOME CLASSES OF OPERATORS ON $C^{*}$-ALGEBRAS
}

\author{
Martin Mathieu
}

\begin{abstract}
This article contains a survey on representation theorems for various classes of linear operators on $C^{*}$-algebras emphasizing the role of local multipliers to derive them.
\end{abstract}

\section{Introduction.}

In studying a class of, perhaps bounded linear, operators on $C^{*}$-algebras one can, at least, distinguish three different levels of understanding this class. Firstly, there are (canonical) examples and necessary properties to be found which guarantee the richness of, and stimulate interest in, this class of operators and help to single out those not contained. Secondly, one seeks to establish sufficient properties that allow to characterize the members of this class of operators in a non-trivial way. Thirdly, and this we should consider the top level of understanding, one aims to determine prototypical examples and a mechanism to build all other members in this class from these. This then enables us in principle to read off all the properties that one is investigating from the prototypical, simple examples.

An instance of such a study arises in the theory of *-derivations of $C^{*}$-algebras. In the first place, these are defined

1991 Mathematics Subject Classification. Primary 47-02; Secondary $46 \mathrm{~L} 57,47 \mathrm{~A} 67,47 \mathrm{~B} 48$.

The support by the European Commission through a Marie Curie Fellowship during the academic year 1997/98 is gratefully acknowledged. 
purely algebraically but turn out to be automatically continuous, a well-known result by Sakai, which has been extended by different methods by several others in each case exploiting interesting properties of $C^{*}$-algebras. Prominent examples are the inner derivations originating from commutators (with self-adjoint elements), thus revealing the intimate interplay with quantum physics. The second step consists in showing that *-derivations precisely are the generators of (norm-continuous) groups of *-automorphisms of $C^{*}$-algebras. The latter are the models of reversible evolutions of quantum systems. But the breakthrough is done via a representation theorem which tells us that every *-derivation is inner, at least for all von Neumann algebras or in some generalized sense.

Our aim here is to discuss a few of such representation theorems for some classes of (not necessarily bounded linear) operators on $C^{*}$-algebras, in particular those recently proven by means of a new tool in operator theory on $C^{*}$-algebras, the local multiplier algebra. We hope to give some flavour of the new ideas involved and to water the mouth for fuller meals, such as offered in [4]. This article comprises two talks delivered to the DIAS Christmas Symposium 1997 in Dublin in December 1997 and the Mathematical Colloquium of the University of Aberdeen in May 1998, respectively.

\section{A few prerequisites.}

In this section we shall recall a few basic notions in the theory of $C^{*}$-algebras that will be relevant for our discussion. Throughout one may think of a $C^{*}$-algebra $A$ as a subalgebra of the bounded linear operators on a Hilbert space, $B(H)$, which is closed in the operator norm and with respect of taking adjoints: if $x \in A$ then so is its adjoint $x^{*}$. The fundamental law that ties together the three basic structures in a $C^{*}$-algebra, the algebraic, the metric, and the order structure, is the $C^{*}$-identity $\left\|x^{*} x\right\|=\|x\|^{2}$ for all $x \in A$. In finite dimensions, the $n \times n$ matrices form a typical example of a $C^{*}$-algebra whereas the first infinite dimensional $C^{*}$-algebra is provided by the compact operators $K(H)$ on a separable Hilbert space $H$. In fact, $K(H)$ is closely related to the matrix algebras as it results from them by taking the direct $\operatorname{limit}_{n} \lim _{n}$ where the 
$n \times n$ matrices $M_{n}$ are embedded into the $(n+1) \times(n+1)$ matrices by putting $a \in M_{n}$ into the upper left hand corner and filling up with zeros. Since $K(H)$ is a closed *-ideal of $B(H)$, the quotient $B(H) / K(H)$ is another prominent example of a $C^{*}$-algebra, the so-called Calkin algebra.

A typical commutative $C^{*}$-algebra is provided by the space $C_{0}(X)$ of all continuous complex-valued functions on a locally compact Hausdorff space $X$ which vanish at infinity. This may be regarded as an algebra of operators by identifying a function with the multiplication by this function on the Hilbert space $L^{2}(X, \mu)$, where $\mu$ gives non-zero measure to every non-empty open subset of $X$, for example. Endowed with these basic examples one can then start to build more complicated ones by a number of procedures, e.g. $C_{0}(X ; K(H))$ where the functions now take on their values in the compact operators rather than the complex numbers but we still use the supremum norm.

One of the constructions with $C^{*}$-algebras that we shall refer to in the sequel is the multiplier algebra of a $C^{*}$-algebra. Supposing that $A$ acts on $H$ non-degenerately, that is, for each $\xi \in H$ which is non-zero there exists an element $x \in A$ such that $x \xi \neq 0$,

$$
M(A)=\{y \in B(H) \mid y A+A y \subseteq A\}
$$

In the examples given above we have $M(K(H))=B(H)$ and $M\left(C_{0}(X)\right)=C_{b}(X)$, the algebra of all bounded continuous functions on $X$. The multiplier algebra $M(A)$ is distinguished by the fact that it is the largest essential extension of $A$, that is, $A$ is contained in $M(A)$ as a closed essential ideal (wherefore it has non-zero intersection with every other non-zero ideal in $M(A)$ ) and every unital $C^{*}$-algebra $B$ that also contains $A$ in the same manner canonically embeds into $M(A)$.

\section{How to apply a representation theorem.}

Before we shall delve into our discussion of some recent representation theorems, let us recall a very familiar one and see how this can be used to obtain some neat consequences. Let $A \subseteq B(H)$, 
$B \subseteq B(K)$ be two $C^{*}$-algebras. A linear mapping $T: A \rightarrow B$ is called bounded if its operator norm

$$
\|T\|=\sup \{\|T x\| \mid x \in A,\|x\| \leq 1\}<\infty .
$$

A requirement which in general is (somewhat) stronger is the following. We say that $T$ is completely bounded if the canonical extension

$$
T \otimes \mathrm{id}: A \otimes_{\min } K\left(\ell^{2}\right) \longrightarrow B \otimes_{\min } K\left(\ell^{2}\right)
$$

is bounded, where the minimal tensor product is obtained from the algebraic tensor product by letting $A \otimes K\left(\ell^{2}\right)$ act canonically on $H \bar{\otimes} \ell^{2}$ and completing in the operator norm. In this case, $\|T\|_{c b}=\|T \otimes \mathrm{id}\|$ is called the cb-norm of $T$.

One of the very nice features of completely bounded operators is that there exists a representation theorem, proved by Paulsen in 1984 .

Theorem. Let $T: A \rightarrow B$ be a completely bounded linear operator between the $C^{*}$-algebras $A$ and $B$. Then there exist a representation $\pi$ of $A$ on a Hilbert space $\hat{H}$ and bounded linear operators $V: \hat{H} \rightarrow K, W: K \rightarrow \hat{H}$ such that $\|V\|\|W\|=\|T\|_{c b}$ and

$$
T x=V \pi(x) W \quad(x \in A) .
$$

This result emerged to be fundamental for the theory of completely bounded operators, for instance, it immediately yields Wittstock's extension and decomposition theorems. For a survey on various aspects of completely bounded operators we refer to [11], and for an up-to-date presentation of their theory in detail to [14].

Completely bounded operators were introduced by Arveson in the late 1960's, and it soon became apparent that it doesn't suffice to study them on $C^{*}$-algebras only. For that reason the notion of an operator space as a subspace $E$ of some $B(H)$ was introduced. Now there arose the question of how to describe these operator spaces abstractly by a neat set of simple axioms, as was done by Gelfand and Naimark for $C^{*}$-algebras. This problem was solved by Ruan in 1988 by providing the following axioms. 
Let $E$ be a complex vector space and let $M_{n}(E)$ denote the vector space of $n \times n$ matrices with entries in $E$. Suppose there exists a sequence $\left(\|\cdot\|_{n}\right)_{n \in \mathbf{N}}$ of norms on $M_{n}(E)$ satisfying the following two requirements

(R1) $\forall n \in \mathbf{N} \forall \alpha, \beta \in M_{n} \forall x \in M_{n}(E)$

$$
\|\alpha x \beta\|_{n} \leq\|\alpha\|\|x\|_{n}\|\beta\|
$$

(R2) $\forall n, m \in \mathbf{N} \forall x \in M_{n}(E) \forall y \in M_{m}(E)$

$$
\|x \oplus y\|_{n+m}=\max \left\{\|x\|_{n},\|y\|_{m}\right\}
$$

Then $\left(E,\left(\|\cdot\|_{n}\right)_{n \in \mathbf{N}}\right)$ is called an abstract operator space. It can be seen easily that, if $E \subseteq B(H)$ and $M_{n}(E)$ is considered as a subspace of $B\left(H^{n}\right)$ canonically, then the above axioms are fulfilled so that, a priori, every operator space is an abstract operator space. Whence the question remains whether the two concepts coincide.

To state Ruan's theorem, which answers this question affirmatively, we have to extend the concept of complete boundedness to the abstract situation. Let $E$ and $F$ be abstract operator spaces. Every linear mapping $T: E \rightarrow F$ yields a linear mapping $T_{n}: M_{n}(E) \rightarrow M_{n}(F)$ by $\left(x_{i j}\right) \mapsto\left(T x_{i j}\right)$. We say that $T$ is completely bounded if each $T_{n}, n \in \mathbf{N}$ is bounded and $\sup _{n}\left\|T_{n}\right\|<\infty$; in that case, this supremum is again called the completely bounded norm $\|T\|_{c b}$ of $T$. (It is not difficult to realize that this gives the same notion as above in the concrete setting.) The operator $T$ is said to be completely isometric if all $T_{n}, n \in \mathbf{N}$ are isometries.

Ruan's Theorem. Every abstract operator space is completely isometrically isomorphic to a subspace of some $B(H)$.

We will now sketch a proof of this fundamental result that relies on another representation theorem related to Paulsen's theorem, taken from [8].

Theorem (Effros-Ruan 1993). Let $E$ be an abstract operator space, and let $f \in M_{n}(E)^{\prime}$ be a bounded linear functional on 
$M_{n}(E)$ (equipped with the norm $\|\cdot\|_{n}$ ). Then there exist a completely bounded linear mapping $\varphi: E \rightarrow M_{n}$ with $\|\varphi\|_{c b} \leq 1$ and bounded linear mappings $v: \mathbf{C}^{n^{2}} \rightarrow \mathbf{C}, w: \mathbf{C} \rightarrow \mathbf{C}^{n^{2}}$, both with norm at most 1 , such that

$$
f(x)=v \varphi_{n}(x) w \quad\left(x \in M_{n}(E)\right) .
$$

Suppose that $E$ is an abstract operator space, and take $x \in M_{n}(E)$. By the Hahn-Banach theorem, there is some $f \in$ $M_{n}(E)^{\prime}$ with the properties $\|f\|=1$ and $|f(x)|=\|x\|_{n}$. By the Effros-Ruan theorem above, there is a complete contraction $\varphi$ from $E$ into $M_{n}$ such that $\left\|\varphi_{n}(x)\right\|=\|x\|_{n}$. Let $\Phi_{n}$ denote the set of all complete contractions from $E$ into $M_{n}$. Letting

$$
\rho: E \longrightarrow \prod_{n \in \mathbf{N}} \prod_{\varphi \in \Phi_{n}} M_{n}
$$

with $\rho(x)=(\varphi(x))$ be the linear mapping that assigns to $x$ the family of all possible evaluations of some $\varphi \in \Phi_{n}, n \in \mathbf{N}$ at $x$, we obtain a complete contraction $\rho$ which, in fact, is completely isometric since there is at least one such $\varphi$ with $\left\|\varphi_{n}(x)\right\|=\|x\|_{n}$. The huge product on the right hand side is a $C^{*}$-algebra, thus sits (completely isometrically) inside some $B(H)$ by the GelfandNaimark theorem, and the proof of Ruan's theorem is complete.

\section{Some classes of operators.}

The following classes of operators have been studied over the past few years by means of multipliers which are defined on ideals of a $C^{*}$-algebra rather than on the entire $C^{*}$-algebra itself.

- elementary operators

- derivations

- Jordan homomorphisms

- Lie derivations, Lie isomorphisms

- centralizing and commuting mappings, both linear and quadratic ones;

bi-derivations 
- commutativity preserving mappings

- orthogonality preserving mappings

- spectrum preserving mappings?

The "?" included into the last item is to indicate that this may be a future project rather than a solved problem. An outstanding question raised by Kaplansky asks whether every unital surjective linear mapping between unital $C^{*}$-algebras preserving the spectrum of every element has to be a Jordan isomorphism. At the time of this writing, this question has been recently resolved in the affirmative for finite von Neumann algebras by Aupetit [5] but was still open in the general case and, following our experience in similar situations, it may be possible that the theory of local multipliers may help to extend the result to general $C^{*}$-algebras.

Rather than attempting an exhaustive discussion of all of the classes of operators listed above, two sample representation theorems will be describe which have been obtained by two of my PhD students lately.

A linear mapping $\theta: A \rightarrow A$ on a $C^{*}$-algebra $A$ is said to be commutativity preserving if it maps commutative subsets of $A$ into commutative subsets, i.e.,

$$
\theta(x) \theta(y)=\theta(y) \theta(x) \quad \text { whenever } \quad x y=y x .
$$

The interest in such mappings stems partially from the fact that these are the mappings that preserve joint measurability of quantum mechanical observables. Of course, such mappings need not be bounded. Some natural examples are provided by multiplications with central elements, mappings into the centre, and, less trivial, surjective Jordan homomorphisms, that is, linear mappings preserving the Jordan product $x y+y x$. Among the first results on this class of operators is a complete description in the case of complex $n \times n$ matrices, $M_{n}$, due to Watkins, Beasley, and Pierce obtained in the late 1970's for $n \geq 3$. Examples show that no such description is possible in the case of $M_{2}$, while $n=1$ of course is trivial.

The following neat theorem obtained by Ralf Banning in 1998 unifies and extends the previously known results, in particu- 
lar the case of von Neumann algebras which was treated by Brešar and Miers in 1993 [7].

Theorem [6]. Let $A$ be a unital boundedly centrally closed $C^{*}$-algebra such that the ideal $K_{2}$ is essential. Let $\theta$ be a bijective linear mapping on $A$ such that both $\theta$ and $\theta^{-1}$ are commutativity preserving. Then there exist a unique invertible element $c$ in the centre $Z(A)$ of $A$, a unique Jordan automorphism $\varphi$ on $A$, and a unique linear mapping $\zeta: A \rightarrow Z(A)$ such that

$$
\theta(x)=c \varphi(x)+\zeta(x) \quad(x \in A) .
$$

The hypothesis 'boundedly centrally closed' will be explained in the subsequent Section 5. The assumption ' $K_{2}$ essential' results from certain polynomial identities that $M_{2}$ satisfies but no matrix algebra $M_{n}$ for higher $n$. These polynomial identities prevent a complete description in the $2 \times 2$ case and thus have to be ruled out. One equivalent formulation is that the set of those irreducible representations of $A$ with dimension at most two has empty interior. The proof of the above theorem heavily relies on a representation theorem for quadratic commuting mappings related to a corresponding result for linear commuting mappings obtained in $[2]$.

The next representation theorem deals with operators that preserve a more geometric property. Let $A \subseteq B(H)$ be a $C^{*}$-algebra. Two elements $x, y \in A$ are said to be orthogonal if $x^{*} y=0$. In geometric terms this means that the ranges $x H$ and $y H$ are orthogonal subspaces of $H$. A linear mapping $T: A \rightarrow B$ between $C^{*}$-algebras $A$ and $B$ is called orthogonality preserving if $T x$ and $T y$ are orthogonal whenever $x$ and $y$ are orthogonal. Immediate examples are ${ }^{*}$-homomorphisms and right multiplications.

The following beautiful representation theorem was proven by Jürgen Schweizer in his thesis in 1996.

Theorem [15]. Let $T: A \rightarrow B(K)$ be a bounded orthogonality preserving operator from the $C^{*}$-algebra $A$ into some $B(K)$. Then there is a representation $\pi$ of $A$ on $K$ and an operator $h \in B(K)$ 
such that

$$
T x=\pi(x) h \quad(x \in A) .
$$

There is an immediate and surprising consequence.

Corollary. Every bounded orthogonality preserving operator is completely bounded.

In none of the above representation theorems is the use of local multipliers apparent; but this is due to the smooth formulation that we chose. In Banning's theorem, the central element $c$ actually arises as a central multiplier of the ideal $K_{2}$, and only the assumption of bounded central closedness brings it back into $A$. All the work in constructing the mappings $\varphi$ and $\zeta$ would not be possible without the use of local multipliers. In Schweizer's theorem, the *-homomorphism in fact maps into the multiplier algebra of the hereditary $C^{*}$-subalgebra generated by $T A$ and the element $h$ is a multiplier of it, at least if $T$ is self-adjoint. Schweizer's methods involve a lot of non-commutative topology, and the representation theorem itself reverts back to an understanding of non-commutative analogues of partially defined continuous mappings. The above theorem indeed is only a special case of a much farther ranging result for Hilbert $C^{*}$-modules, as the product $x^{*} y$ may already indicate.

\section{Local multipliers and how to use them.}

The local multiplier algebra $M_{\mathrm{loc}}(A)$ of a $C^{*}$-algebra $A$ is the direct limit (in the category of $C^{*}$-algebras) of the multiplier algebras $M(I)$ of closed essential ideals $I$ in $A$. The connecting *-homomorphisms are given by the restriction of multipliers on an essential ideal $I$ to the essential ideal $J$ if $J \subseteq I$. This construction, which was first pursued by Elliott and Pedersen in the mid 1970's [9], [13], resembles closely the construction of the symmetric ring of quotients of a semiprime ring à la Kharchenko $[\mathbf{1 0}]$, and in fact, it is the exact $C^{*}$-analogue of it. There are also close interconnections between the algebraic construction, denoted by $Q_{s}(A)$, and $M_{\mathrm{loc}}(A)$ : the bounded part of $Q_{s}(A)$ (in the order theoretic sense of Handelman-Vidav), $Q_{b}(A)$, is dense in $M_{\text {loc }}(A)$ 
and conversely, $Q_{s}(A)$ is the central localization of $Q_{b}(A)$. This interplay has been thoroughly studied by Pere Ara and myself since the early 1990's and will be laid out in [4]. One of the fundamental results is the local Dauns-Hofmann theorem describing the centre of $M_{\mathrm{loc}}(A)$ in a similar fashion as the Dauns-Hofmann theorem does for the centre of the multiplier algebra $M(A)$.

Theorem [1]. Let $A$ be a $C^{*}$-algebra. Then the centre $Z\left(M_{\mathrm{loc}}(A)\right)$ of $M_{\mathrm{loc}}(A)$ is an $A W^{*}$-algebra, and its structure space is the inverse limit (in the category of compact spaces) over the Stone-Čch compactifications of all open dense subsets of the primitive spectrum of $A$.

Being an $A W^{*}$-algebra, $Z\left(M_{\mathrm{loc}}(A)\right)$ is rich in projections, and this allows to some extent to replace the use of the spectral theorem in von Neumann algebras. The local Dauns-Hofmann theorem also gives rise to the concept of boundedly centrally closed $C^{*}$-algebras as follows. Among its consequences is the fact that the centre of the local multiplier algebra of $M_{\mathrm{loc}}(A)$ coincides with $Z\left(M_{\mathrm{loc}}(A)\right)$, which parallels perfectly the algebraic situation. (It is not known whether or not $M_{\mathrm{loc}}\left(M_{\mathrm{loc}}(A)\right)$ coincides with $M_{\text {loc }}(A)$, however.) Denoting by ${ }^{c} A$ the $C^{*}$-algebra $\overline{A Z\left(M_{\mathrm{loc}}(A)\right)}$, the bounded central closure of $A$, this entails that ${ }^{c}\left({ }^{c} A\right)={ }^{c} A$ wherefore $A \mapsto{ }^{c} A$ is a proper closure operation. A $C^{*}$-algebra $A$ thus is said to be boundedly centrally closed if $A={ }^{c} A$. There are several equivalent formulations of this property each of which is quite useful: $Z(M(A))=Z\left(M_{\mathrm{loc}}(A)\right)$; the primitive spectrum of $A$ is extremally disconnected; $A$ is self-injective as a Banach $A$-bimodule. Examples of boundedly centrally closed $C^{*}$-algebras are many, such as von Neumann algebras, more generally $A W^{*}$-algebras, prime $C^{*}$-algebras, hereditary $C^{*}$-subalgebras of boundedly centrally closed ones, ...

The way one should think of the transition from $A$ to its bounded central closure ${ }^{c} A$, or to $M_{\text {loc }}(A)$ possibly, is in analogy to the transition from $A$ to its enveloping von Neumann algebra $A^{\prime \prime}$. The advantage is, however, that we do not need to refer to weak topologies (and hence stay inside the category of $C^{*}$-algebras throughout) as the closure operation is performed by an algebraic 
plus norm closure procedure. The traditional approach in operator theory on $C^{*}$-algebras to appeal to $A^{\prime \prime}$, and hence to von Neumann algebras, is as follows. In studying a class of operators one first restricts attention to the much nicer class of von Neumann algebras and derives the properties under this additional assumption. Then ones uses that, for every bounded linear operator $T$ on a $C^{*}$-algebra $A$, the second adjoint $T^{\prime \prime}$ provides a canonical bounded extension to $A^{\prime \prime}$ so that one may apply the previously obtained results in the more general setting. This route is taken, for instance, in [15]. It has the disadvantages that the operators necessarily have to be bounded and that the extension $T^{\prime \prime}$, in general, may not inherit the properties that $T$ had.
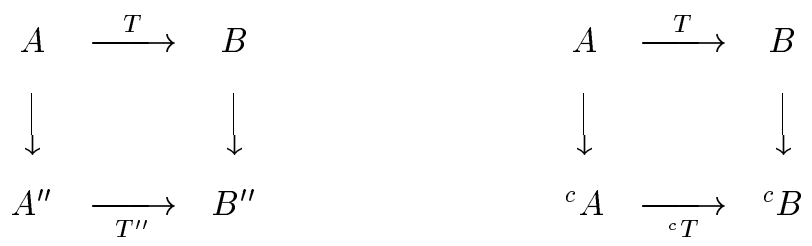

The new approach, via local multipliers, suggests to study operators first on the class of boundedly centrally closed $C^{*}$-algebras and then extend them to the bounded central closure. This has the advantage of being much more algebraic and often simpler than the traditional path; [6] is an example of a rather fruitful application of this approach.

Another one is the following nice result describing the norm of an inner derivation on a $C^{*}$-algebra. Suppose that $a$ is an element in the multiplier algebra $M(A)$ of a $C^{*}$-algebra $A$. By $\delta_{a}$ we shall denote the linear mapping on $A$ defined by $x \mapsto x a-a x$, $x \in A$. This is called an inner derivation, for it satisfies the Leibniz product rule

$$
\delta_{a}(x y)=x\left(\delta_{a} y\right)+\left(\delta_{a} x\right) y \quad(x, y \in A) .
$$

It is immediate that the norm $\left\|\delta_{a}\right\|$ is estimated above by $2 d(a, Z(M(A))$, the distance of $a$ from the centre $Z(M(A))$. Since the late 1960's, starting with work by Kadison, Lance and 
Ringrose, many authors studied situations in which actual equality holds (which in general fails). For example, equality holds for von Neumann algebras, and this was extended to boundedly centrally closed $C^{*}$-algebras in $[\mathbf{1 2}]$. Following the 'new approach' as in [12], and combining it with the result in [16] stating that the distance to the centre of a $C^{*}$-algebra is always attained, we derive the following result.

Theorem. Let $\delta_{a}, a \in M(A)$ be an inner derivation on the $C^{*}$-algebra $A$. Then there exists a local multiplier $b$ of $A$ such that $\delta_{a}=\delta_{b}$ and $\left\|\delta_{b}\right\|=2\|b\|$.

By means of this, the norm of an inner derivation is computed precisely for an arbitrary $C^{*}$-algebra. An extension of this result to generalized inner derivations can be found in [4].

The success in applying local multipliers to study operators on $C^{*}$-algebras of course motivated further investigation of the local multiplier algebra itself. In particular the ideal structure of $M_{\mathrm{loc}}(A)$ needs to be understood better. One of the surprises in these recent studies was the result that there exist non-simple $C^{*}$-algebras for which the local multiplier algebra can be simple, in striking contrast to the situation of the global multiplier algebra $M(A)[\mathbf{3}]$.

\section{References}

1. P. Ara and M. Mathieu, A local version of the Dauns-Hofmann theorem, Math. Z. 208 (1991), 349-353.

2. P. Ara and M. Mathieu, An application of local multipliers to centralizing mappings of $C^{*}$-algebras, Quart. J. Math. Oxford (2) 44 (1993), 129-138.

3. P. Ara and M. Mathieu, A simple local multiplier algebra, Math. Proc. Cambridge Phil. Soc. 125 (1999), in press.

4. P. Ara and M. Mathieu, "Local multipliers of $C^{*}$-algebras", in preparation.

5. B. Aupetit, Sur les transformations qui conservent le spectre, in: E. Albrecht and M. Mathieu (eds.), "Banach Algebras '97", Walter de Gruyter, Berlin 1998; 55-78. 
6. R. Banning, Kommutativitätserhaltende Abbildungen auf $C^{*}$-Algebren, Doctoral Thesis, Univ. of Tübingen, Tübingen, 1998.

7. M. Brešar and C. R. Miers, Commutativity preserving mappings of von Neumann algebras, Can. J. Math. 45 (1993), 695-708.

8. E. G. Effros and Zh.-J. Ruan, On the abstract characterization of operator spaces, Proc. Amer. Math. Soc. 119 (1993), 579-584.

9. G. A. Elliott, Automorphisms determined by multipliers on ideals of a $C^{*}$-algebra, J. Funct. Anal. 23 (1976), 1-10.

10. V. K. Kharchenko, "Automorphisms and derivations of associative rings", Kluwer Acad. Publ., Dordrecht, 1991.

11. M. Mathieu, Derivations and completely bounded maps on $C^{*}$-algebras. A survey, Irish Math. Soc. Bull. 26 (1991), 1741.

12. M. Mathieu, The cb-norm of a derivation, in: R. E. Curto and P. E. T. Jørgensen (eds.), "Algebraic methods in operator theory", Birkhäuser, Basel, 1994; 144-152.

13. G. K. Pedersen, Approximating derivations on ideals of $C^{*}$-algebras, Invent. math. 45 (1978), 299-305.

14. G. Pisier, "Similarity problems and completely bounded maps", LNM 1618, Springer, Berlin 1996.

15. J. Schweizer, Interplay between noncommutative topology and operators on $C^{*}$-algebras, Doctoral Thesis, Univ. of Tübingen, Tübingen, 1996.

16. D. W. B. Somerset, The proximinality of the centre of a $C^{*}$-algebra, J. Approx. Theory 89 (1997), 114-117.

Martin Mathieu

Department of Pure Mathematics,

The Queen's University of Belfast

Belfast BT7 1NN

email: m.m@qub.ac.uk 